\title{
Double differential distribution of electron emission in the ionization of water molecules by fast bare oxygen ions
}

\author{
Shamik Bhattacharjee ${ }^{1}$, Shubhadeep Biswas ${ }^{1}$, Chandan Bagdia ${ }^{1}$, \\ Madhusree Roychowdhury ${ }^{1}$, Saikat Nandi ${ }^{1}$, Deepankar Misra ${ }^{1}$, J M Monti $^{2}$, \\ C A Tachino ${ }^{2}$, R D Rivarola ${ }^{2}$, C Champion $^{3}$ and Lokesh C Tribedi ${ }^{1}$ \\ ${ }^{1}$ Tata Institute of Fundamental Research, Homi Bhabha Road, Colaba, Mumbai 400005, India \\ ${ }^{2}$ Laboratorio de Colisiones Atómicas, Facultad de Ciencias Exactas, Ingenieriía y Agrimensura, and \\ Instituto de Fiísica Rosario (CONICET-UNR), Avenida Pellegrini 250, 2000 Rosario, Argentina \\ ${ }^{3}$ Centre dEtudes Nuclaires de Bordeaux Gradignan (CENBG), CNRS/IN2P3, Université Bordeaux 1, \\ Chemin du Solarium, BP120, 33175 Gradignan, France \\ E-mail: lokesh@tifr.res.in
}

Received 19 June 2015, revised 6 January 2016

Accepted for publication 7 January 2016

Published 23 February 2016

\begin{abstract}
The doubly differential distributions of low-energy electron emission in the ionization of water molecules under the impact of fast bare oxygen ions with energy of $48 \mathrm{MeV}$ are measured. The measured data are compared with two quantum-mechanical models, i.e. the post and prior versions of the continuum distorted wave-eikonal initial state (CDW-EIS) approximation, and the first-order Born approximation with initial and final wavefunctions verifying correct boundary conditions (CB1). An overall excellent qualitative agreement is found between the data and the CDW-EIS models whereas the CB1 model showed substantial deviation. However, the detailed angular distributions display some discrepancies with both CDW-EIS models. The single differential and total cross-sections exhibit good agreement with the CDW-EIS models. The present detailed data set could also be used as an input for modeling highly charged ion induced radiation damage in living tissues, whose most abundant component is water. Similar measurements are also carried out for a projectile energy of $60 \mathrm{MeV}$. However, since the double differential cross-section data show similar results the details are not provided here, except for the total ionization cross-sections results.
\end{abstract}

Keywords: ion collision with molecules, water molecule, e-spectroscopy, experiment, radiation damage, CDW-EIS model, low-energy electron DDCS spectrum

(Some figures may appear in colour only in the online journal)

\section{Introduction}

The importance of water, which makes up about $60 \%$ of the weight of our body, is quite obvious and the research of all aspects of this molecule is of great interest in many branches of physics. In the case of molecules, ionization processes carry the signature of the multi-center character of the target wavefunction. Various inelastic processes for atomic targets [1-3], such as binary collision mechanisms, two-center effects and soft collision mechanisms, also influence the results of ion-molecule collisions. The systematics of low-energy electron emission from a homo-nuclear diatomic molecule under the impact of charged particles are being studied using $\mathrm{H}_{2}$ [4-6], $\mathrm{O}_{2}$ [7-9] and $\mathrm{N}_{2}[10,11]$ as targets to investigate the Young-type electron interference. As water is one of the simplest among all tri-atomic molecules, its ionization under impact from highly charged ions could thus provide benchmark results towards understanding the fundamentals of relevant interaction mechanisms. Particularly with the increasing use of very high $(\mathrm{GeV})$ energy heavy ions, 
including protons, as a means of radiotherapy [12], it is necessary to determine the exact effects created by these charged particles while passing through living organisms. The heavy ions (such as protons and carbon nuclei) are used to provide an efficient way to treat the affected cells by a significant increase of the dose profile at the end of the particle range, i.e. in the so-called Bragg peak region [13]. In a worldwide effort to build a collisional database of interest for radiation therapy using fast heavy ions, experiments are being carried out to measure the ion induced fragmentation and ionization of relevant bio-molecules. For instance, in the case of RNA-base molecules, the total ionization cross-sections (TCS) under impact from various bare and dressed projectiles [14-16] as well as protons with energies of a few $\mathrm{keV}$ $[17,18]$ to a few $\mathrm{MeV}[19,20]$ have been reported recently. The interactions of highly charged ions with a solid medium and clusters are, however, more complicated. In addition, the interaction of fast ions with biological tissues leads to the production of a large number of secondary electrons. These low-energy electrons can initiate further ionization and excitation processes which may lead to single and double strand breaks of the DNA molecule [21]. This means that in order to exactly determine the damage induced in radiation therapy, we require microscopic knowledge of the collisional events which happened during the passage of heavy ions. It thus becomes very important to obtain an accurate description of the TCSs and differential cross-sections for the ionization of water molecules (serving as a model for biological matter) induced by highly charged ions, to measure the probability of occurrence of these inelastic processes.

Despite its great significance, measurements of differential cross-sections and TCSs for the emission of low-energy electrons from water molecules are sparsely reported in contemporary research. These experimental studies are mostly limited to electrons [22-24], protons [25-27] and $\alpha$-particles [28-30] as projectiles. In the case of electron induced ionization of water molecules, the experimental measurements were found to be in very good agreement with the calculations performed in the framework of the distorted wave Born approximation [31]. For the ionization of water vapor under $\mathrm{MeV}$ energy proton impacts, measurements were well reproduced by various quantum-mechanical treatments. Different theoretical models within the framework of the Born approximation were employed in order to evaluate the influence of each pairwise Coulomb interaction term among the ejected electron, the scattered proton and the residual ionized target in the final state [32]. Another approach involving separation of collision dynamics and molecular geometry has also accurately reproduced the experimental data for $\mathrm{p}-\mathrm{H}_{2} \mathrm{O}$ collisions [33]. Similarly, for $\mathrm{MeV}$ energy $\alpha$-particle impact, the data were found to be in good quantitative agreement with the predictions of the FBA-CW model where the incident and scattered particles are described by a plane wave and the ejected electron is described by a Coulomb wave [34]. So far, the only measurement of electron double differential crosssections (DDCS) as well as single differential cross-sections (SDCSs) for ionization of water molecules using highly charged ions has been carried out with $\mathrm{C}^{6+}, \mathrm{O}^{8+}$ and $\mathrm{He}^{2+}$ ions [30, 35, 36]. However, the measured cross-sections (TCS as well as DDCS) using bare carbon ions are observed to be largely lower than the theoretical calculations [36], whereas the DDCS data for bare $\mathrm{O}$ ions are reasonably well reproduced by the continuum distorted wave-eikonal initial state (CDW-EIS) model [35]. It is important to extend these measurements to different projectiles with different energies in order to have a better and complete understanding of the theoretical models as well as for the creation of a broad data base.

In the present investigation, we have measured the absolute DDCS, SDCS and TCS for the ionization of water molecules for two different beam energies. The experimental data have been compared with both the prior and post versions of the CDW-EIS approximation as well as the first-order Born approximation with initial and final wavefunctions satisfying correct boundary conditions (CB1). Atomic units are used in the following, unless mentioned otherwise.

\section{Experimental details}

\subsection{Apparatus}

The experiments were performed using $\mathrm{O}^{8+}$ ions, with two different incident energies, namely, $48 \mathrm{MeV}$ and $60 \mathrm{MeV}$. The ion beams were available from the $14 \mathrm{MV}$ Pelletron accelerator facility at TIFR, Mumbai. In brief, the interaction chamber is made of a stainless steel cylinder of 18 inched in diameter and 15 inches high. The electrostatic spectrometer is made of two hemispherical analyzers with its inner and outer electrodes having radii of 2.5 and $3.5 \mathrm{~cm}$, respectively [37]. These analyzers and the housing were made of oxygen free high conductivity copper. The spectrometer is situated on top of a motorized turn table used to choose different scattering angles. About 200 c.c. of de-ionized water was kept in a vacuum sealed container at room temperature. It was connected to the gas inlet of the scattering chamber for the water molecules from the container to enter in the vapor phase, followed by a solenoid valve for controlling the rate of flow at a fixed pressure. This was made possible by the fact that the vapor pressure of water at room temperature (taken to be 27 ${ }^{\circ} \mathrm{C}$ ) is about 27 Torr. The base pressure in the interaction chamber was maintained constantly at around $2 \times 10^{-7}$ Torr using a $3000^{-1}$ turbo-molecular pump. The experiments were carried out with the scattering chamber uniformly filled with water vapor, at a pressure of 0.15 mTorr. The secondary electrons ejected in the ion-molecule collision processes were initially energy analyzed by the spectrometer. For this purpose suitable voltages were applied to both electrodes of the spectrometer. These electrons were then detected using a channel electron multiplier (CEM), which was placed at the exit slit of the analyzer.

The CEM was used in counting mode. The front of the CEM was kept at $100 \mathrm{~V}$ to increase the detection efficiency of low-energy electrons. The final output pulse from the tail of the CEM was connected to a constant fraction discriminator (CFD) via a coupling capacitor. The TTL pulse from the CFD 

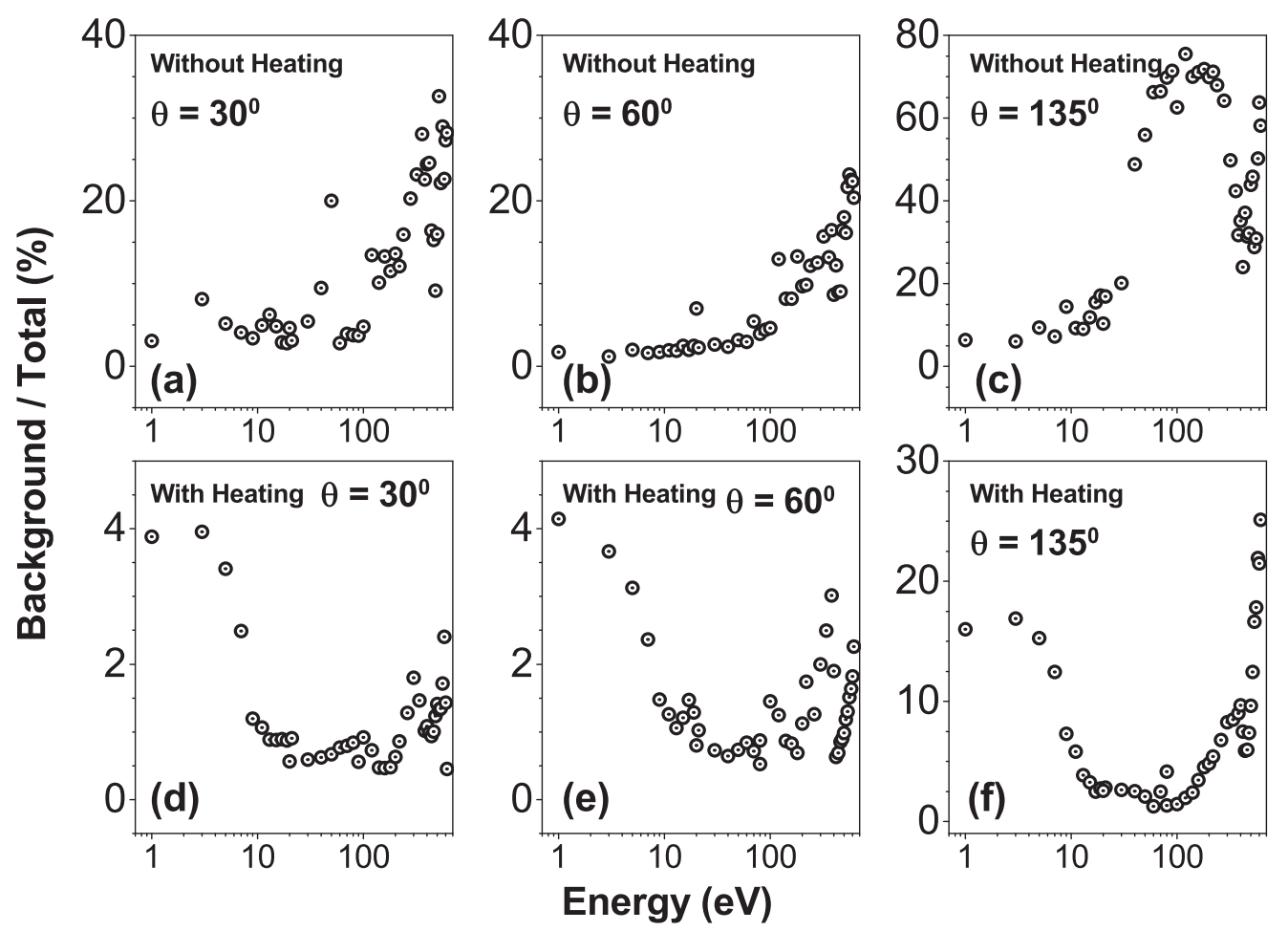

Figure 1. (a)-(f) The background fraction, i.e. background-to-total-count ratio, for $48 \mathrm{MeV} \mathrm{O}^{8+}$ projectiles. (a)-(c) represent the fraction without heating the chamber while (d)-(f) represent the fraction with heating for emission angles of $30^{\circ}, 60^{\circ}$ and $135^{\circ}$. It can be seen that with the heater on, the background fraction is reduced considerably in (d)-(f).

output was fed to the counter input of the LabView based interface.

At each ejection angle, the electrons emitted with different energies during the interactions were counted for an amount of incident projectile charge collected on a Faraday cup. Extreme precaution was taken regarding cleanliness and minimizing any stray electric field by avoiding any insulating surface inside and by using oil-free pumps. Two layers of $\mu$ metal were used inside the chamber to shield it from the Earth's magnetic field.

\subsection{Measurement of the double differential cross-sections}

The DDCSs at a given emission angle, were obtained from the first principle by measuring all the required experimental parameters, i.e. beam current, gas pressure inside the chamber and the spectrometer dimensions. The efficiency of the spectrometer was typically $5 \%$ of the electron energy. In the CEMer experiment [35], the background to data ratio was found to be too high due to the sticking of the water vapor to the inner wall of the chamber and subsequent re-evaporation. For example, at high electron energies, i.e. above $100 \mathrm{eV}$, the background was as high as $15 \%-50 \%$ (or even $60 \%$ at a few energies) of the total count (figure 1). So the experiment was repeated again with the focus of keeping the background much lower. The cylindrical surface of the chamber was heated and kept at a temperature of about $40{ }^{\circ} \mathrm{C}$. The temperature inside the chamber rapidly falls to $34^{\circ} \mathrm{C}$ and then remains constant in the rest of the central area containing the collision and detection zone, thereby not affecting the CEM performance [38]. The temperature in the center of the chamber was higher than room temperature only by about $7^{\circ}$ $\mathrm{K}$ causing gas density reduction by a few percent only. The water container was, however, kept at room temperature and thereby the vapor pressure inside the container was unaffected. The wall heating reduced the sticking of water molecules on the chamber wall and hence reduced the background to about $2 \%-5 \%$ of the data below $100 \mathrm{eV}$ and a maximum of about $10 \%-12 \%$ at higher energies. Figure 1 shows the improvement of the background fraction, i.e. the background-count to total-count ratio, by heating the scattering chamber during the experiment. Figure 1 shows the background fraction for the case of $48 \mathrm{MeV}$ data. This is an improvement over our previous similar experiment with $72 \mathrm{MeV} \mathrm{O}^{8+}$ on water molecules [35].

The electron DDCS spectra were collected for eleven emission angles, namely, $20^{\circ}, 30^{\circ}, 45^{\circ}, 60^{\circ}, 75^{\circ}, 80^{\circ}, 90^{\circ}$, $105^{\circ}, 120^{\circ}, 135^{\circ}$ and $150^{\circ}$ (figure 2). At each angle the emitted electrons were detected over a large energy range, starting from as low as $1 \mathrm{eV}$, and ending at $600 \mathrm{eV}$. As discussed above, the background counts $N_{\mathrm{b}}$, obtained in the absence of the target gas, were small $\left(\sim 2 \%-12 \%\right.$ of $\left.N_{\mathrm{e}}\right)$. Overall, the error due to statistical fluctuation was measured to be less than $5 \%$ for all of the emission angles. The uncertainty in the target gas pressure was 7\%. Subsequently, the maximum absolute error in the DDCS was found to be about $20 \%$ which includes the uncertainty in efficiency $(\sim 10 \%)$, resolution $(\sim 8 \%-10 \%)$ and solid-angle estimation ( $\sim 10 \%$ ) or any small change in the density during heating of the chamber. 


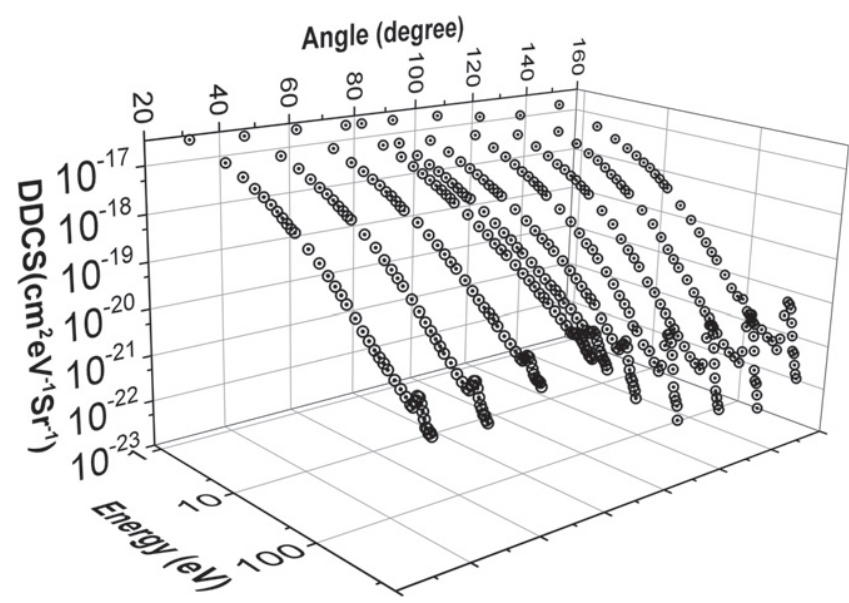

Figure 2. The 3D plot showing the energy distribution of the DDCS measured at all the angles for $48 \mathrm{MeV} \mathrm{O}^{8+}$ projectile.

\section{Theoretical models}

\subsection{Continuum distorted wave-eikonal initial state approximation}

In the CDW-EIS approximation for single ionization under the impact of a bare ion, an independent electron model is often employed. In this model a multi-electronic target is usually reduced to a mono-electronic one, assuming that the rest of the electrons remain frozen in their initial orbitals. In the entrance channel the initial bound state of the active electron is described by means of a Hartree-Fock wavefunction distorted by a multiplicative eikonal phase. The latter represents the active electron in a continuum state of the projectile Coulomb field. Similarly, in the exit channel the continuum of the active electron is described by the product of a plane wave and two continuum factors, each one of them associated with the residual target and projectile fields $[39,40]$. Thus the model inherently takes into account the effect of both target and projectile centers (two-center effect; see the next section) into the exit channel. One may use the perturbative potentials either in the initial channel distorted wavefunction or in the final channel distorted one, invoking the notion of the prior and post versions of the transition matrix element, $T_{\text {if }}^{-}$and $T_{\text {if }}^{+}$respectively. They can expressed as follows:

$$
T_{\text {if }}^{-}=\left\langle\chi_{\mathrm{f}}^{-}\left|W_{\mathrm{i}}\right| \chi_{\mathrm{i}}^{+}\right\rangle
$$

and

$$
T_{\text {if }}^{+}=\left\langle\chi_{\mathrm{f}}^{-}\left|W_{\mathrm{f}}^{\dagger}\right| \chi_{\mathrm{i}}^{+}\right\rangle
$$

respectively. Here $\chi_{\mathrm{i}}^{+}$and $\chi_{\mathrm{f}}^{-}$represents the initial and final channel distorted wavefunctions, as discussed above. $W_{\mathrm{i}}$ is the EIS perturbative operator in equation (1), while in equation (2) $W_{\mathrm{f}}$ is the CDW perturbative operator [41].

The contribution arising from the potential for the multielectronic target in the exit channel is commonly approximated by that of an effective Coulombic one $\left(V_{\mathrm{T}}\right)$ between the active electron and the target nucleus carrying an effective charge. It is set as: $V_{\mathrm{T}}(\vec{r}) \simeq-Z_{\mathrm{T}}^{\text {eff }} / r$. Here, the position of the active electron in a reference frame fixed on the target is represented by $\vec{r}$. The quantity $Z_{\mathrm{T}}^{\text {eff }}$ is the effective target charge chosen to preserve the binding energy $\varepsilon_{\mathrm{i}}$ of the active electron in its initial orbital. In general, the relation between the effective charge and the binding energy is given by $Z_{\mathrm{T}}^{\text {eff }}=n_{\mathrm{i}} \sqrt{-2 \varepsilon_{\mathrm{i}}}$, with $n_{\mathrm{i}}$ being the principal quantum number of the initial orbital. Thus, when using this approach the interaction of the passive electrons with the active electron is only considered via an effective Coulombic interaction. This description of the active-electron continuum is commonly used in both versions of the CDW-EIS model to facilitate the calculations. More complete numerical continuum wavefunctions have also been used in previous works [42]. In the prior version and for atomic multi-electronic targets, the perturbation operator is applied on a distorted wavefunction where a Roothaan-Hartree-Fock description is used for the initial bound state, which includes radial electronic correlation. In the post version, in the final perturbation potential, a term including the residual non-Coulombic interaction between the passive and active electrons, known as 'dynamic screening', was in general omitted in the calculations.

Consequently, a discrepancy arises between the crosssection values calculated by both prior and post versions of the CDW-EIS model. As expected the inclusion of this residual potential could lead to entirely diminished post-prior discrepancies $[43,44]$. In the present calculations we exclude the dynamic screening term in order to estimate its influence on the cross-sections. For molecular targets, as for the case considered here, an additional approximation arises from the description of the structure of the molecular orbitals. We have chosen to describe the initial state within the complete neglect of differential overlap (CNDO) approximation, where the molecular orbitals are expressed in terms of atomic ones corresponding to the atomic constituents. We must mention that for $\mathrm{H}_{2} \mathrm{O}$ molecules it has been shown that CDW-EISDDCSs as a function of the emission angle, averaged over all possible molecular orientations, are almost insensitive to the description of the target initial wavefunction [45]. Finally, the DDCS as a function of the emitted electron energy $\left(E_{\mathrm{e}}\right)$ and solid angle $\left(\Omega_{\mathrm{e}}\right)$ are obtained by an integration on the momentum transfer of the square modulus of the transition matrix element in either version of the CDW-EIS model.

\subsection{CB1 approximation}

The cross-sections have been also calculated within the firstorder Born approximation with initial and final wavefunctions verifying $\mathrm{CB} 1$. This approach can be interpreted as an extension to the case of the ionization of molecular targets of the CB1 model introduced by Belkić et al [46, 47] for studying the electron capture from atomic targets. In order to represent the multi-centered nature of the target $\left(\mathrm{H}_{2} \mathrm{O}\right.$ molecule in vapor phase), the electronic populations of the target have been described by means of molecular orbitals constructed from a linear combination of atomic orbitals in a selfconsistent field approximation [48]. In this approach, the molecular orbitals were expressed in terms of Slater functions all centered at a common origin coinciding with the oxygen 
nucleus. Thus, providing suitable analytical wavefunctions is quite similar to the atomic case, in which the basis set consists of functions all referred to a common origin. In the laboratory framework, the triple differential cross-sections (TDCS), differential in the direction of the scattered projectile $\mathrm{d} \Omega_{\mathrm{s}}$, in the direction of the ejected electron $\mathrm{d} \Omega_{\mathrm{e}}$ and in the ejected energy $\mathrm{d} E_{\mathrm{e}}$, denoted in the following by $\sigma^{3}\left(\Omega_{\mathrm{s}}, \Omega_{\mathrm{e}}, E_{\mathrm{e}}\right)$, are defined as [34],

$$
\begin{aligned}
\sigma^{(3)}\left(\Omega_{\mathrm{s}}, \Omega_{\mathrm{e}}, E_{\mathrm{e}}\right) & \equiv \frac{\mathrm{d}^{3} \sigma}{\mathrm{d} \Omega_{\mathrm{s}} \mathrm{d} \Omega_{\mathrm{e}} \mathrm{d} E_{\mathrm{e}}}=\sum_{j=1}^{N_{\mathrm{s}}} \frac{\mathrm{d}^{3} \sigma_{j}}{\mathrm{~d} \Omega_{\mathrm{s}} \mathrm{d} \Omega_{\mathrm{e}} \mathrm{d} E_{\mathrm{e}}} \\
& =\sum_{j=1}^{N_{\mathrm{s}}} 16 M_{\mathrm{p}}^{2} Z_{\mathrm{p}}^{2} \frac{k_{1} k_{\mathrm{s}}}{k_{\mathrm{i}}}\left|\left[T_{\mathrm{if}}\right]_{j}\right|^{2},
\end{aligned}
$$

where the transition matrix element between the initial state labeled ' $\mathrm{i}$ ' and the final state labeled 'f', denoted as $\left[T_{\mathrm{if}}\right]_{j}$, is expressed by

$$
\left[T_{\mathrm{if}}\right]_{j}=\left\langle\Psi_{\mathrm{f}}^{j}\left(\mathbf{r}_{0}, \mathbf{r}_{1}\right)\left|V\left(\mathbf{r}_{0}, \mathbf{r}_{1}\right)\right| \Psi_{\mathrm{i}}^{j}\left(\mathbf{r}_{0}, \mathbf{r}_{1}\right)\right\rangle .
$$

In equation (3), $\mathbf{k}_{\mathrm{i}}, \mathbf{k}_{\mathrm{s}}$ and $\mathbf{k}_{1}$ represent the wave vectors of the incident projectile, scattered projectile and ejected electron, respectively. $M_{\mathrm{p}}$ denotes the mass of a proton and $N_{\mathrm{s}}=5$ is the number of sub-shells in the water molecule. Here, $Z_{\mathrm{p}}$ denotes the nuclear charge on the bare projectile $\left(Z_{\mathrm{p}}=8\right.$ in this case). Now, by using the well-known 'frozen core' approximation which reduces the present ten-electron problem to a one-active-electron problem, the interaction potential $V\left(\mathbf{r}_{0}, \mathbf{r}_{1}\right)$ can be simply written as,

$$
V\left(\mathbf{r}_{0}, \mathbf{r}_{1}\right)=-\frac{Z_{\mathrm{p}}}{\left|\mathbf{r}_{0}-\mathbf{r}_{1}\right|}+\frac{Z_{\mathrm{p}}}{\left|\mathbf{r}_{0}\right|},
$$

where $\mathbf{r}_{1}$ is the position vector of the active electron with respect to the framework center and $\mathbf{r}_{0}$ is the position vector of the incident projectile with respect to the framework center.

Finally, the DDCSs are obtained by analytical integration of the TDCSs $\sigma^{3}\left(\Omega_{\mathrm{s}}, \Omega_{\mathrm{e}}, E_{\mathrm{e}}\right)$ over the scattered projectile direction [50]. It may be mentioned here that the Moccia's wavefunctions [49] are obviously provided for a given target orientation. Under these conditions and aiming at comparing the theoretical predictions with the experiment, the calculations are here performed by using an average orientation of the water target. This averaging is performed by means of the rotation operator applied to each molecular sub-shell, that leads to a simple analytical expression of the transition matrix.

\section{Results and discussion}

\subsection{Double differential cross-sections}

4.1.1. Energy distribution. Figure 2 shows a threedimensional plot representing the measured energy dependence of the DDCS for all the angles for an impact energy of $48 \mathrm{MeV}$. However, to avoid confusion the theoretical calculations are not shown in this plot. Such a

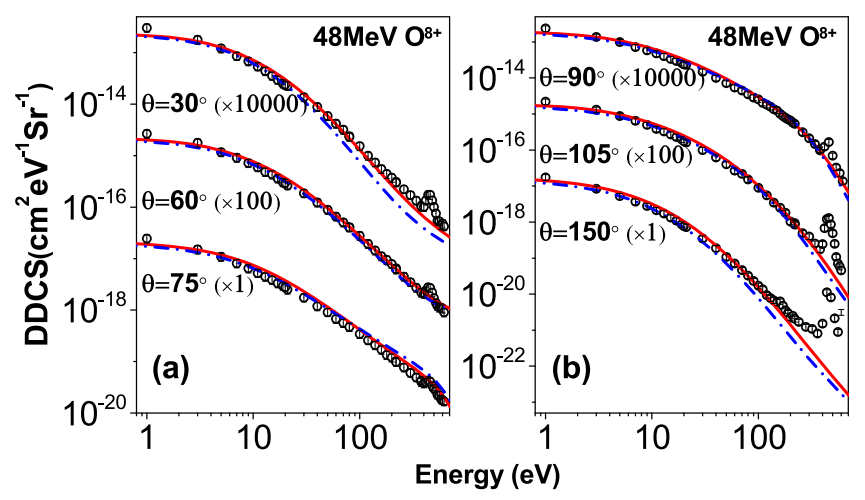

Figure 3. The absolute electron DDCSs for different emission angles for the collision systems $48 \mathrm{MeV} \mathrm{O}^{8+}+\mathrm{H}_{2} \mathrm{O}$ (a), (b). The solid (dashed) red (blue) lines correspond to the model predictions of the CDW-EIS prior (post) version. The comparisons are shown for six different angles.

comparison is displayed in figure 3 for selected angles and for both the impact energies.

The measured energy distributions of the absolute $\mathrm{e}^{-}$ DDCS are shown in figure 3 for both $48 \mathrm{MeV} \mathrm{O}^{8+}$ projectiles. In both cases, the cross-section decreases over several orders of magnitude with the increase in electron energy, at each emission angle. At $30^{\circ}$ it falls of by four orders of magnitude, whereas it decreases by five orders in the case of $150^{\circ}$. The sharp peak around $480 \mathrm{eV}$ in each plot corresponds to the target-oxygen KLL-Auger electron emission. The experimental data were compared with the theoretical calculations using the CDW-EIS model for both the prior and post versions of the scattering matrix, using the CNDO approximation to represent the initial state. In general, a good qualitative agreement between the theory and the experimental data is obtained for different electron emission angles.

The agreement with the CDW-EIS (prior) model is very good for all the angles, at least qualitatively. A closer inspection reveals some deviations of the prior model from the experimental data at energies between $10 \mathrm{eV}$ and $50 \mathrm{eV}$ for $75^{\circ}$ and $90^{\circ}$ (see figures 3 (a), (b)). A good quantitative agreement can be seen for energies between $80 \mathrm{eV}$ and $200 \mathrm{eV}$, for all emission angles. As far as the CDW-EIS (post) model is concerned, the agreement is again nearly perfect except for the high-energy part for $30^{\circ}$ and $150^{\circ}$ for which the predictions fall a little above $50 \mathrm{eV}$. Similarly, for $60 \mathrm{MeV}$ $\mathrm{O}^{8+}$, both the prior and post versions again give a very good qualitative agreement with the data (not shown) throughout the entire energy range. It should be noted that the quantitative agreement between the data and the CDW-EIS prior version is better compared to that for the post version only for extreme forward and backward angles. For the rest of the angles, both these models give nearly perfect agreement. The prior-version better conforms with experiment for large scattering angles because it includes the influence of dynamic screening. The difference between them may be attributed to the influence of the dynamic screening. This observation is a bit different than that for the higher energy $(72 \mathrm{MeV})$ data [35] for the same projectile ions. The line spectrum KLL- 


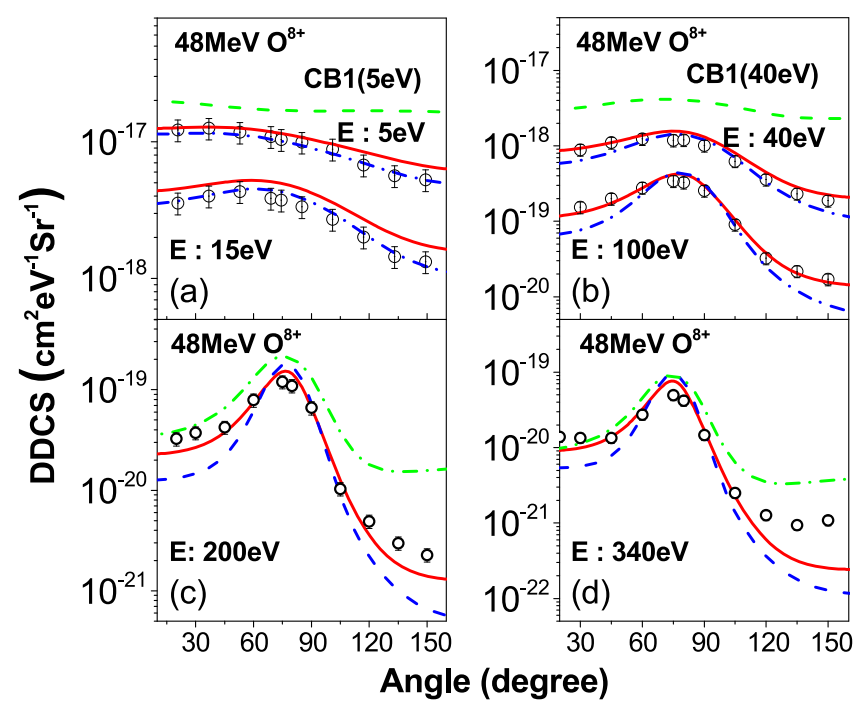

Figure 4. The angular dependence of the DDCS for different electron energies for $48 \mathrm{MeV}$ projectiles. The solid (dashed) red (blue) line corresponds to the CDW-EIS prior (post) version. The green dasheddotted line corresponds to theoretical calculations involving the CB1 approximations.

Auger electron is not relevant for comparison with the models here.

4.1.2. Angular distributions. Since the DDCS values fall by three to four orders of magnitude in the previous plots (figure 3), it may be difficult to explore the finer details regarding the discrepancy between experiment and theory from such plots in logarithmic scales. In this regard, the angular distributions at fixed emission energies are useful quantities in order to make a stringent test of the models. Figures 4(a)-(d) shows the angular distributions of the absolute electron DDCSs at different ejected electron energies for a beam energy of $48 \mathrm{MeV}$. For higher energy electrons, the distributions gradually become sharply peaked around $75^{\circ}$. The difference in the shape of the distributions for low- and high-energy electrons is due to the well-known binary nature of collisions [51, 52]. Overall, the prior and post versions of the CDW-EIS approximation have qualitatively reproduced the shape of the experimental data. It can easily be seen that the CB1 model largely deviates from the data except for the high energy, i.e. $340 \mathrm{eV}$, for which the agreement is only good for forward angles (see figure 4). The predictability of the CB1 model is therefore quite poor. Considering that CB1 is a one-center (target-one) approximation, its failure could be attributed to the necessity of using descriptions where the emitted electron evolves in the simultaneous presence of the projectile and target fields, as in the CDW-EIS model. We must take into account that the distortions in the initial and final channels associated with the projectile-activeelectron interaction implicitly open higher orders of the Bornseries and avoid the possible existence of singularities associated with disconnected diagrams.

On the other hand, a very good quantitative agreement between the data for $48 \mathrm{MeV}$ (figures 4(a)-(d)) and the prior version of the CDW-EIS approximation can be seen for all the ejected electron energies over the entire angular region, except for 200 and $340 \mathrm{eV}$ for which the agreement is a bit poor for higher backward angles (see figure 4). The post version of the CDW-EIS model generally underestimates the data for backward and forward angles for all emission energies. In fact, both the prior and post versions agree excellently with the data in the mid-angular region (i.e. $60^{\circ}-$ $90^{\circ}$ ) over which the binary collision mechanism has stronger contribution. It may be instructive to compare these results with those obtained for a simple helium atom in collisions with bare $\mathrm{F}$ ions with a similar velocity, i.e. for an energy of $4 \mathrm{MeV} \mathrm{u}^{-1}$ (see figure 4 of [3]). In the case of He atoms the agreement with the CDW-EIS model was excellent for the angular distributions for all the energies between 5 and $300 \mathrm{eV}$. The deviation for large backward angle was observed only above $300 \mathrm{eV}$ (for example see the data for $400 \mathrm{eV}$ in figure 4(h) of [3]. The agreement for the low forward angles as well as large backward angles was better in the case of helium than that for the water molecule (see figure 5(a)-(h)). However, these comparisons are only indicative since the collision systems are not identical in the two cases.

\subsection{Single differential cross-sections}

4.2.1. Energy distribution. The variation in the SDCS spectra over different ejected energies can be obtained by integrating the angular distribution of the DDCS over the solid angle of electron emission $\Omega_{\mathrm{e}}$,

$$
\frac{\mathrm{d} \sigma}{\mathrm{d} E_{\mathrm{e}}}=\int_{\theta_{\mathrm{i}}}^{\theta_{\mathrm{f}}} \frac{\mathrm{d}^{2} \sigma}{\mathrm{d} \Omega_{\mathrm{e}} \mathrm{d} E_{\mathrm{e}}} \mathrm{d} \Omega_{\mathrm{e}} .
$$

Figure 5(a) shows the energy distribution of the SDCS values for the impact energy $48 \mathrm{MeV}$. In figure 5(c) we display the TCS, as discussed below. The DDCS values have been integrated over an angular range of $\theta=20^{\circ}$ to $\theta=150^{\circ}$ to obtain the $\frac{\mathrm{d} \sigma}{\mathrm{d} E_{\mathrm{e}}}$ values. Overall, the energy dependence of the distribution has been qualitatively very well reproduced by both theoretical models. However, as far as the absolute agreement is concerned, the post version exhibits slightly better agreement with the data (see the inset) considering the error-bars. The ratio between the SDCS data and the post version, as well as that with the prior version, are shown in the inset in figure 5(a). Some deviation in the ratio from the unity is noticed between 5 and $200 \mathrm{eV}$.

4.2.2. Angular distribution. The dependence of the SDCS values over the electron emission angle can be obtained by integrating the energy distributions of the DDCS spectra over the ejected electron energy $E_{\mathrm{e}}$ as

$$
\frac{\mathrm{d} \sigma}{\mathrm{d} \Omega_{\mathrm{e}}}=\int_{\epsilon_{\mathrm{i}}}^{\epsilon_{\mathrm{f}}} \frac{\mathrm{d}^{2} \sigma}{\mathrm{d} \Omega_{\mathrm{e}} \mathrm{d} E_{\mathrm{e}}} \mathrm{d} E_{\mathrm{e}} .
$$

We have shown the angular distribution of the SDCS for the ejected electrons in figure 5(b). The experimental DDCS values have been numerically integrated over an energy range 

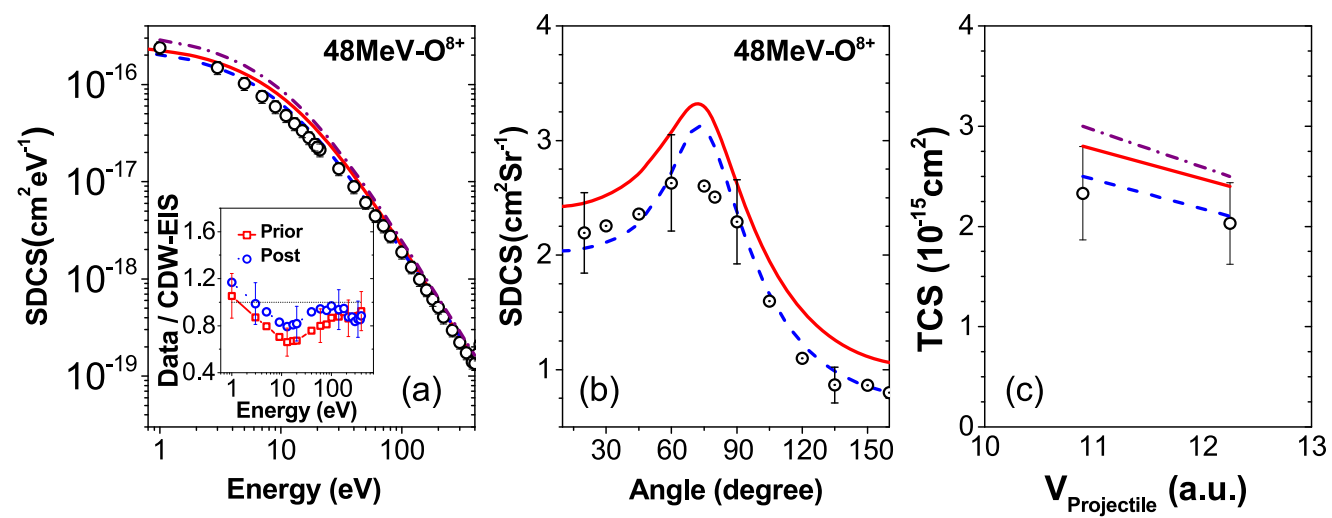

Figure 5. (a) Energy distribution of the electron SDCS. (b) Angular distribution of SDCS $\mathrm{d} \sigma / \mathrm{d} \Omega$. (c) TCS versus energy along with theoretical models. The solid red (dashed blue) line in each plot corresponds to the theoretical calculations using the prior (post) version of the CDW-EIS model. The dashed-dotted line correspond to Rudd's model. The inset in figure 5(a) displays the ratio between the SDCS data and the post (blue-circle) version, as well as the prior version (red-square).

Table 1. TCSs in units of $10^{3} \mathrm{Mb}$.

\begin{tabular}{lcccc}
\hline$E(\mathrm{MeV})$ & Exp. & CDW-EIS prior & CDW-EIS post & Rudd model \\
\hline 48 & $2.33(45)$ & 2.8 & 2.5 & 3.0 \\
60 & $2.03(35)$ & 2.4 & 2.1 & 2.5 \\
\hline
\end{tabular}

of $1-400 \mathrm{eV}$. Although the overall shape of the experimental SDCS spectra resembles those of the theoretical models, quantitative discrepancies between the former and the latter can be seen. The CDW-EIS post version reproduces the experimental distribution quite well (figure 5(b)), whereas the prior version overestimates the data. The difference between the SDCS $(\mathrm{d} \sigma / \mathrm{d} \Omega)$ data for the two energies (48 and $60 \mathrm{MeV})$ are small compared to the absolute uncertainties in the data and hence the data for $60 \mathrm{MeV}$ are not shown.

4.2.3. The Rudd model. We have calculated $\mathrm{d} \sigma / \mathrm{d} \epsilon$ using Rudd's semi-empirical approach [53], which was initially developed for providing a parameter-based description of the electron emission spectrum from water vapor after proton impact. Despite being a semi-empirical relation, the Rudd's model provides remarkably close agreement with experimental SDCS data, figure 5. Although it overestimates the low-energy data by about $30 \%-70 \%$, at higher energy, i.e. above $50 \mathrm{eV}$, the model comes closer to the data, i.e. within $20 \%$.

\subsection{Total cross-sections}

The TCSs are obtained by numerically integrating the experimental $\mathrm{d} \sigma / \mathrm{d} \Omega$ values over the entire angular range from $0^{\circ}-180^{\circ}$. The experimental values for the SDCS at emission angles smaller than $20^{\circ}$ and larger than $150^{\circ}$ were extrapolated from the obtained data using the fact that the SDCS is flat near $0^{\circ}$ and $180^{\circ}$. The value of the extrapolated cross-section was about $7 \%$ of the TCS.

The experimental TCSs for different projectile energies (E) are tabulated in table 1 and are shown in figure 5(c). As can be seen, the TCS values calculated using the CDW-EIS (post and prior) approximation, are quite close to the data, i.e. overestimating only by $10 \%-25 \%$, respectively, at $48 \mathrm{MeV}$, and by $5 \%-20 \%$ at $60 \mathrm{MeV}$. Rudd's model overestimates the data by about $30 \%$ and $25 \%$, respectively, for the $48 \mathrm{MeV}$ and $60 \mathrm{MeV}$ projectiles. Finally, it may be noted that the deviations of both the CDW-EIS models from the experimental TCS are within the experimental error-bars. Another notable point is the slightly better agreement of the post version with the TCS and SDCS data (figure 5) over the prior model. These results show slight variation to those presented for a $72 \mathrm{MeV} \mathrm{O}^{8+}+\mathrm{H}_{2} \mathrm{O}$ collision system [35].

\section{KLL-Auger cross-sections of oxygen}

In figures 3(a)-(b) sharp Auger emission peaks can be seen for all the measured angles. The distribution shows an almost flat behavior which normally one expects in the case of $1 \mathrm{~s}$ ionization in ion-atom collisions. These data were integrated to obtain the total inner shell ionization cross-section for both the projectiles. The obtained TCS values are 6.1 and $6.6 \mathrm{Mb}$, respectively, for 48 and $60 \mathrm{MeV}$ ions.

\section{Conclusions}

The absolute DDCS, SDCS and TCS for electron emission in the ionization of water molecules under the impact of bare oxygen ions have been measured. The energy and angular distribution of the DDCS and the derived values of the SDCS are used to provide a detailed stringent test for a number of theoretical models, such as ab initio CDW-EIS (post and prior versions), CB1 and Rudd's semi-empirical model. The energy 
Table 2. e-DDCS (in $\mathrm{Mb}$ ) for $48 \mathrm{MeV}$ bare $\mathrm{O}$ ions on water.

\begin{tabular}{lccccccccccc}
\hline$E$ & $30^{\circ}$ & $45^{\circ}$ & $60^{\circ}$ & $75^{\circ}$ & $80^{\circ}$ & $90^{\circ}$ & $105^{\circ}$ & $120^{\circ}$ & $135^{\circ}$ & $150^{\circ}$ & $\frac{\mathrm{d} \sigma}{\mathrm{d} \epsilon_{\mathrm{e}}}$ \\
\hline 1 & 30.546 & 26.578 & 26.693 & 24.551 & 24.139 & 23.694 & 22.0840 & 15.662 & 12.836 & 17.491 & 238.842 \\
5 & 12.180 & 12.549 & 11.709 & 10.883 & 10.387 & 9.908 & 8.836 & 6.761 & 5.647 & 5.273 & 102.119 \\
9 & 6.821 & 7.0910 & 7.169 & 6.454 & 6.391 & 5.818 & 4.975 & 3.549 & 3.0461 & 2.793 & 58.853 \\
20 & 2.384 & 2.853 & 2.936 & 2.787 & 2.649 & 2.357 & 1.750 & 1.195 & 0.910 & 0.774 & 22.651 \\
40 & 0.885 & 1.101 & 1.232 & 1.183 & 1.194 & 1.0093 & 0.624 & 0.357 & 0.231 & 0.187 & 8.845 \\
60 & 0.420 & 0.530 & 0.657 & 0.673 & 0.686 & 0.540 & 0.267 & 0.121 & 0.0775 & 0.0704 & 4.448 \\
80 & 0.236 & 0.308 & 0.406 & 0.470 & 0.443 & 0.352 & 0.152 & 0.0613 & 0.0359 & 0.0326 & 2.758 \\
100 & 0.153 & 0.199 & 0.278 & 0.341 & 0.325 & 0.254 & 0.0902 & 0.0323 & 0.0217 & 0.0170 & 1.885 \\
120 & 0.0998 & 0.132 & 0.193 & 0.259 & 0.239 & 0.180 & 0.0556 & 0.0214 & 0.0125 & 0.00939 & 1.323 \\
140 & 0.0740 & 0.0935 & 0.144 & 0.207 & 0.186 & 0.135 & 0.0348 & 0.0131 & 0.00868 & 0.00672 & 0.987 \\
160 & 0.0550 & 0.0702 & 0.111 & 0.170 & 0.151 & 0.102 & 0.0252 & 0.00905 & 0.00572 & 0.00630 & 0.768 \\
180 & 0.0441 & 0.0539 & 0.0948 & 0.134 & 0.131 & 0.0786 & 0.0181 & 0.00629 & 0.00445 & 0.00358 & 0.615 \\
200 & 0.0372 & 0.0422 & 0.0789 & 0.119 & 0.109 & 0.0660 & 0.0103 & 0.00491 & 0.00297 & 0.00227 & 0.509 \\
220 & 0.0306 & 0.0330 & 0.0635 & 0.100 & 0.0887 & 0.0534 & 0.00891 & 0.00346 & 0.00237 & 0.00205 & 0.415 \\
260 & 0.0222 & 0.0249 & 0.0446 & 0.0768 & 0.0708 & 0.0314 & 0.00490 & 0.00244 & 0.00149 & 0.00146 & 0.297 \\
300 & 0.0170 & 0.0171 & 0.0349 & 0.0605 & 0.0524 & 0.0211 & 0.00399 & 0.00161 & 0.00108 & 0.00115 & 0.222 \\
340 & 0.0134 & 0.0133 & 0.0271 & 0.0493 & 0.0418 & 0.0145 & 0.00251 & 0.00126 & 0.00094 & 0.00094 & 0.173 \\
380 & 0.0104 & 0.0110 & 0.0219 & 0.0412 & 0.0338 & 0.0101 & 0.00175 & 0.00089 & 0.00086 & 0.00116 & 0.138 \\
\hline $\mathrm{d} \sigma$ & 225.432 & 241.662 & 261.926 & 259.0377 & 250.836 & 214.457 & 159.551 & 109.801 & 86.370 & 86.0406 & 2373 \\
\hline $\mathrm{d} \Omega$ & & & & & & & & & & &
\end{tabular}

dependence of the DDCS values show excellent overall qualitative agreement with the post and prior versions of the CDW-EIS model with some deviations for high-energy electrons emitted in small forward and large backward angles. The CB1 model, however, gives a large deviation from the measured data. The energy dependence of the SDCS values are also in quantitative agreement with the theoretical calculations using the CDW-EIS model and to some extent with Rudd's semi-empirical one. The angular and energy dependence of the SDCS is, however, slightly better reproduced by the post version of the CDW-EIS model. The absolute TCSs are also well reproduced by the CDW-EIS (post and prior) models within experimental errors, the post model being slightly closer to the data. The present observation regarding the good agreement of the measurements with the CDW-EIS model along with the CNDO approximation will provide useful input for further modeling of the radiation damage of water by oxygen or heavy ions of similar atomic numbers with energies of a few $\mathrm{MeV} \mathrm{u}^{-1}$. However, more investigations are required using projectiles with higher atomic numbers and high charge states in order to test the validity of the models on a wide range of ions and energies.

\section{Acknowledgments}

We would like to thank the Pelletron Accelerator Group for smooth operation of the machine. JMM, CAT and RDR acknowledge the Agencia Nacional de Promoción Cientiífica y Tecnológica de la República Argentina for financial support throughout the project PICT 2011-2145.

\section{References}

[1] Stolterfoht N, Platten H, Schiwietz G, Schneider D, Gulyás L, Fainstein P D and Salin A 1995 Phys. Rev. A 523796

[2] Tribedi L C, Richard P, Wang Y D, Lin C D, Gulyás L and Rudd M E 1998 Phys. Rev. A 583619

[3] Misra D, Kelkar A H, Fainstein P D and Tribedi L C 2012 J. Phys. B: At. Mol. Opt. Phys. 45225201

[4] Tribedi L C, Richard P, Wang Y D, Lin C D and Olson R E 1996 Phys. Rev. Lett. 773767

[5] Stolterfoht N et al 2001 Phys. Rev. Lett. 87023201

[6] Misra D, Kadhane U, Singh Y P, Tribedi L C, Fainstein P D and Richard P 2004 Phys. Rev. Lett. 92 153201

[7] Winkworth M, Fainstein P D, Galassi M E, Baran J L, Dassanayake B S, Das S, Kayani A and Tanis J A 2009 Nucl. Instrum. Methods Phys. Res. B 267373

[8] Nandi S, Agnihotri A N, Kasthurirangan S, Kumar A, Tachino C A, Rivarola R D, Martiín F and Tribedi L C 2012 Phys. Rev. A 85062705

[9] Nandi S, Agnihotri A N, Tachino C A, Rivarola R D, Martiín F and Tribedi L C 2012 J. Phys. B: At. Mol. Opt. Phys. 45215207

[10] Baran J L, Das S, Járái-Szábo F, Póra K, Nagy L and Tanis J A 2008 Phys. Rev. A 78012710

[11] Tachino C A, Martiín F and Rivarola R D 2011 J. Phys. B: At. Mol. Opt. Phys. 45025201

[12] Schardt D, Elsässer T and Schulz-Ertner D 2010 Mod. Phys. Rev. 82383

[13] Amaldi U and Kraft G 2005 Rep. Prog. Phys. 681861

[14] Agnihotri A N et al 2012 Phys. Rev. A 85032711

[15] Agnihotri A N, Kasthurirangan S, Nandi S, Kumar A, Galassi M E, Rivarola R D, Champion C and Tribedi L C 2013 Phys. Rev. A 87032716

[16] Agnihotri A N et al 2013 J. Phys. B: At. Mol. Opt. Phys. 46 185201

[17] Moretto-Capelle P and Le Padellec A 2006 Phys. Rev. A 74 062705 
[18] Tabet J, Eden S, Feil S, Abdoul-Carime H, Farizon B, Farizon M, Ouaskit S and Märk T D 2010 Phys. Rev. A 82 022703

[19] Iriki Y, Kikuchi Y, Imai M and Itoh A 2011 Phys. Rev. A 84 032704

[20] Itoh A, Iriki Y, Imai M, Champion C and Rivarola R D 2013 Phys. Rev. A 88052711

[21] Boudaïfa B, Cloutier P, Hunting D, Huels M A and Sanche L 2000 Science 2871658

[22] Schutten J, de Heer F J, Moustafa H R, Boerboom A J H and Kistemaker J 1966 J. Chem. Phys. 443924

[23] Märk T D and Egger F 1976 Int. J. Mass Spectrosc. Ion Phys. 2089

[24] Bolorizadeh M A and Rudd M E 1986 Phys. Rev. A 33882

[25] Toburen L H and Wilson W E 1977 J. Chem. Phys. 665202

[26] Rudd M E, Goffe T V, Dubois R D and Toburen L H 1985 Phys. Rev. A 31492

[27] Bolorizadeh M A and Rudd M E 1986 Phys. Rev. A 33888

[28] Toburen L H, Wilson W E and Popowich R J 1980 Radiat. Res. 8227

[29] Rudd M E, Goffe T V and Itoh A 1985 Phys. Rev. A 322128

[30] Ohsawa D, Sato Y, Okada Y, Shevelko V P and Soga F 2005 Phys. Rev. A 72062710

[31] Champion C, Hanssen J and Hervieux P A 2002 J. Chem. Phys. 117197

[32] Boudrioua O, Champion C, Dal Cappello C and Popov Y V 2007 Phys. Rev. A 75022720

[33] Lüdde H J, Spranger T, Horbatsch M and Kirchner T 2009 Phys. Rev. A 80 060702(R)

[34] Champion C, Boudrioua O, Dal Cappello C, Sato Y and Ohsawa D 2007 Phys. Rev. A 75032724
[35] Nandi S, Biswas S, Khan A, Monti J M, Tachino C A, Rivarola R D, Misra D and Tribedi L C 2013 Phys. Rev. A 87052710

[36] Dal Capello C, Champion C, Boudrioua O, Lekadir H, Sato Y and Ohsawa D 2009 Nucl. Instrum. Methods B 267781

[37] Misra D, Thulasiram K V, Fernandes W, Kelkar A H, Kadhane U, Kumar A, Singh Y P, Gulyas L and Tribedi L C 2009 Nucl. Instrum. Methods Phys. Res. B 267157

[38] See, for example, www.sjuts.com/CEMModels-Standard.html

[39] Crothers D S F and McCann J F 1983 J. Phys. B: At Mol. Phys. 163229

[40] Fainstein P D, Ponce V H and Rivarola R D 1988 J. Phys. B: At. Mol. Opt. Phys. 21287

[41] Fainstein P D, Ponce V H and Rivarola R D 1991 J. Phys. B: At. Mol. Opt. Phys. 243091

[42] Fainstein P D, Gulyas L and Salin A 1994 J. Phys. B 27 L259

[43] Monti J M, Fojón O A, Hanssen J and Rivarola R D 2010 J. Phys. B: At. Mol. Opt. Phys. 43205203

[44] Monti J M, Fojón O A, Hanssen J and Rivarola R D 2013 J. Phys. B: At. Mol. Opt. Phys. 46145201

[45] Tachino C A, Monti J M, Fojón O A, Champion C and Rivarola R D 2014 J. Phys. B 47035203

[46] Belkić D 1978 J. Phys. B: At. Mol. Opt. Phys. 113529

[47] Belkić D, Gayet R, Hanssen J and Salin A 1986 J. Phys. B: At. Mol. Opt. Phys. 192945

[48] Champion C 2003 Phys. Med. Biol. 482147

[49] Moccia R 1964 J Chem. Phys. 402186

[50] Champion C 2010 Phys. Med. Biol. 5511

[51] Bonsen T F M and Vriens L 1970 Physica 47307

[52] Manson S T, Toburen L H and Stolterfoht N 1975 Phys. Rev. A 1260

[53] Rudd M E 1989 Nucl. Tracks Radiat. Meas. 16213 